\title{
The Reliability Analysis of RC Beams after High Temperature Based on the Layered Method
}

\author{
Xindong ZHAO \\ School of civil engineering, Xian University of \\ Architecture and Technology, Xi'an, China \\ E-mail: zhaoxdgh@xauat.edu.cn \\ Yunlei SU \\ School of civil engineering, Xian University of \\ Architecture and Technology, Xi'an, China \\ E-mail: 574096771@qq.com
}

\author{
Tao BAI \\ School of civil engineering, Xian University of \\ Architecture and Technology, Xi'an, China \\ E-mail: baitao1990@126.com \\ Yijie DING \\ School of civil engineering, Xian University of \\ Architecture and Technology, Xi'an, China \\ E-mail: 462355962@qq.com
}

\begin{abstract}
This paper calculated the reduction factors with layered method for beam sections' intensity after fire condition. With these factors, it built up a resistance model of reinforced concrete construction members. By analyzing the statistical parameters of resistance in statistics, and considering the random of this model, it calculated the reliability of $\mathrm{RC}$ beams with improving the checking point method.
\end{abstract}

Keywords-the layered method; reduction factors; resistance model; improved checking point method

\section{INTRODUCTION}

After suffering from high temperature in the fire, the mechanical properties of materials in the reinforced concrete structures will have the degradation of different degree, leading to the decline of the component even the decrease of the bearing capacity of the whole structure. In order to investigate the reliability of the structure after fire, the calculation of residual load bearing capacity of reinforced concrete structures after high temperature is necessary. Base on the theory and experience of the past researches, after high temperature for reinforced concrete structure and the components, the principle and the method to calculate the bearing capacity is as same as the room temperature calculation. While, the material deformation, strength and other related parameters should be made some correction, based on the distribution of section temperature field $[1,2,3]$. The purpose of this paper is to discuss the calculation method of the reinforced concrete beam member's bearing capacity after high temperature preliminarily.

Among the calculation methods of the residual load bearing capacity of reinforced concrete structures after high temperature, there are mainly the equivalent section method and the layered method. The equivalent section method assumes the strength of the steel before and after high temperature is constant, but the dimensions will be cut, then the reduction factor of the component's intensity can be calculated. The layered method divides component into different units, and make a weighted average of the intensity to get the reduction factor. So, it reflects intensity of the components after high temperature actually. Therefore, the layered method is selected to calculate the reduction factor of the components[4,5].

\section{THE LAYERED METHOD}

In the layered method, to compensate the defects for the uneven distribution of temperature field within component interface which causes the different component's intensity, it divides the component into different units after fire, then, the reduction factor is got through a weighted average of the intensity by the finite difference principle[6].

\section{A. The Assumptions of the Layered Method}

When the residual load bearing capacity of reinforced concrete structures after high temperature is calculated, the assumptions are developed as follows:

- The component's intensity of every unit is a constant after the pressed concrete is meshed;

- The equivalent reduction factor of concrete intensity in compression is got by the weighted average of the compressive areas;

- There is no relative slip between concrete and reinforcing steel bar in the fire;

- The influence of the tensile areas of concrete is ignored, when calculating the residual load bearing capacity;

- The influence on the strength of materials of different temperature - stress path and the temperature stress is not considered in the Components within the cross section.

\section{B. Meshing}

First, the Component cross section is meshed before calculating the residual load bearing capacity of reinforced concrete structures. Because the Concrete is the thermal inertia material, the temperature heat up fast at the Component surface, while it forms a larger temperature gradient In the component shallow depth. So, when the component is meshed In the shallow depth, the finer grid units are selected; while the internal temperature gradient is relatively much smaller, the larger units are selected [7]. 
There is the assume the beam size is $\mathrm{b} \times \mathrm{h}$, the cross section of transverse is divided into $\mathrm{n}$ equal, and the longitudinal section of transverse is divided into m equal. So, the whole cross section is divided into $\mathrm{m} \times \mathrm{n}$ units. Each unit is made in a node number, and the highest temperature of a unit is made $(\mathrm{i}, \mathrm{j})$ as $T_{i, j}$ by a certain fire time, then the center of the unit temperature is on behalf of the entire grid.

\section{The Confirm of the Reduction Factor of the Components}

There is the assume concrete is the isotropic materials. After determining the initial conditions, the boundary conditions and the thermal parameters of material, the cross section of the highest temperature distribution are analysis at different time after the fire by ANSYS, then temperature field is divided into several different areas according to certain temperature difference(usually $50^{\circ} \mathrm{C}--100^{\circ} \mathrm{C}$ ). According to each area of the highest temperature in different fire time, the equivalent reduction factor of concrete intensity $\overline{\varphi_{C T i}}$ of each unit is got by the model. Finally, the whole section is weighted averagely by the layered method to get the equivalent reduction factor of the whole section

$\overline{\varphi_{C T}}$ [8]. The regression formula between concrete strength and temperature after the high temperature is as follows:

- when $\mathrm{T} \leq 200^{\circ} \mathrm{C}$ :

$$
f_{c r}(T)=\left[1.0-0.58194\left(\frac{T-20}{1000}\right)\right] \bullet f_{c}
$$

- when $\mathrm{T} \geq 200^{\circ} \mathrm{C}$ :

$$
f_{c r}(T)=\left[1.1459-1.39255\left(\frac{T-20}{1000}\right)\right] \bullet f_{c}
$$

In the formula, $f_{c r}(T)$ is the compressive intensity of concrete materials after T temperature; $f_{c}$ is the compressive intensity of concrete materials when the T temperature.

$$
\overline{\varphi_{C T i}}=\frac{f_{c r}(T)}{f_{c}}
$$

The formula of the equivalent reduction factor of the whole section $\overline{\varphi_{C T}}$ :

$$
\overline{\varphi_{C T i}}=\frac{\sum \overline{\varphi_{C T i}} \cdot \mathrm{S}_{\mathrm{i}}}{\sum \mathrm{S}_{\mathrm{i}}}
$$

In the formula, $\mathrm{S}_{\mathrm{i}}$ is the area of $\mathrm{i}$ in compression; $\overline{\varphi_{C T i}}$ is the equivalent reduction factor of concrete intensity of $i$, then we can get the equivalent reduction factor of concrete intensity. And, the equivalent reduction factor of steel bar intensity is got by "The reliability analysis of reinforced concrete's structural members after fire". By the way, the remaining bearing capacity of the reinforced concrete beam is got after fire.

\section{The Analysis Method of Statistical Analysis AND RELIABILITY OF THE FACTORS INFLUENCING THE RESISTANCE}

In the present design for building structure, there are two kinds of common resistance calculation, deformation checking and bearing capacity checking. The object of the former calculation can be used for overall structure or single structural components, while the latter checking object only for structure of individual component. In this article, the strength analysis is only for structures, so we only checked bearing capacity. Based on a lot of experimental results, there are varied factors influencing the resistance of structures and there are three significant factors, the uncertainty of geometric parameter $\mathrm{X}_{a}$, the uncertainty of material performance $\mathrm{X}_{m}$ and the uncertainty of computational model $\mathrm{X}_{p}$. The three random variables above is normal distribution, so the structure resistance is a product function containing multiple random variables [9].

\section{A. The Statistical Analysis of The Factors Affecting Component Resistance}

\section{1) The uncertainty of structures material performance}

The ambiguity of structures' material properties mainly refers to raw material for component in terms of ambiguity physical properties. There are varied factors, material production quality, manufacturing technology, deposit technology and the work environment conditions, causing the uncertainty of material.

Set the random variable $\mathrm{X}_{m}$ as uncertainty of component materials, so:

$$
X_{m}=\frac{f_{c}}{k_{o} f_{k}}
$$

Where $f_{c}$ is the actual material performance of structures; $f_{k}$ is the component material standard code stipulated by specifications; $k_{o}$ is the difference coefficient between current structure design codes component material performance and the practice of material performance. The variable material coefficient of variation for the normal distribution is:

$$
\delta_{\mathrm{Xm}}=\sqrt{\delta_{X 0}^{2}+\delta_{f_{s}}^{2}}
$$

2) The uncertainty of component geometry parameter

There are many factors affect structural element whether in the making process of the structures in the early or the final-period installation process, making the geometrical 
characteristics of the structural components (such as geometry, inertia, resisting moment) different from pre design. The differences are defined as the uncertainty of geometry parameter.

The component geometric feature ambiguity is represented by applying random variables, the formula is:

$$
\mathrm{X}_{\mathrm{a}}=\frac{a}{a_{k}}
$$

Where $\mathrm{a}$ is the actual value building set parameters; $a_{k}$ is standard value building set parameters, generally using design value. Then the variable coefficient of variation for the normal distribution is:

$$
\delta_{X_{A}}=\delta_{a}
$$

\section{3) The uncertainty of computational model}

Because the basic assumptions and the certain deviation between actual situation and calculation formula of approximate calculation caused by the uncertainty in the structural resistance calculation, the character is defined as the uncertainty of computational model and described it with the formula:

$$
X_{p}=\frac{R_{0}}{R_{c}}
$$

Where $R_{0}$ is the actual resistance of component; $R_{c}$ is the calculating resistance of component according to the related structural design specification.

Through the relevant statistical analysis in China, some researchers obtained statistical parameters of calculation model uncertainty about reinforced concrete structures. It is shown in tab. 1:

TABLE I. THESTATISTICAL PARAMETER OF THE UNCERTAINTY OF COMPUTATIONAL MODEL $X_{P}$ ABOUT REINFORCED CONCRETE STRUCTURAL ELEMENT

\begin{tabular}{llcc}
\hline \multirow{2}{*}{ Types of structures } & Strained condition & $\mu_{X_{p}}$ & $\delta_{X_{p}}$ \\
& Axial tension & 1.00 & 0.04 \\
\cline { 2 - 4 } & Axial compression & 1.00 & 0.05 \\
\cline { 2 - 4 } $\begin{array}{c}\text { Reinforced concrete } \\
\text { structural element }\end{array}$ & Eccentric Compression & 1.00 & 0.05 \\
\cline { 2 - 4 } & Bending & 1.00 & 0.04 \\
\cline { 2 - 4 } & Shear & 1.00 & 0.15 \\
\cline { 2 - 4 } & &
\end{tabular}

Considering the main influence factors, the resistance model of reinforced concrete structures can be expressed with random variable $\mathrm{R}$.

$$
R=X_{p} R_{p}=X_{p} R\left(f_{c 1} a_{1}, f_{c 2} a_{2}, \cdots, f_{c n} a_{n}\right)
$$

Where $R_{p}$ is the resistance obtained by calculating formula; $\mathrm{R}$ ( )is the function $R_{p} ; f_{c i}$ is the related performance of component material $\mathrm{i} ; a_{i}$ is material set the corresponding components parameters i. Based on the above formula, we can know

$$
R=X_{p} R\left(\mathrm{X}_{m 1} f_{k l} \mathrm{k}_{01} \bullet \mathrm{X}_{A 1} \mathrm{a}_{k 1} \cdots, \mathrm{X}_{m n} f_{k n} \mathrm{k}_{0 n} \bullet \mathrm{X}_{A n} \mathrm{a}_{k n}\right)
$$

Where $X_{p}, X_{m i}, X_{A i}$ respectively represent the random variables of calculation mode, components material, component set parameters.

\section{B. The Improved Checking Point Method of Building Structures Reliability after Fire}

The checking point method is modified on the basis of the center point method which initially put forward by professor Hasofer-laind and Rackwitz-Fiessler. In this method, the non-normal random variables of actual distribution types of information are taken into account, so the reliability index $\beta$ is calculated in more accurate approximation and satisfies the limit state equation with adding a few work[10].

Although the checking point method has been improved a lot compared to the center point method, there is a complex iterative computation when $\mathrm{R}$ or $\mathrm{S}$ are non-normal variables. In the improved checking point method, the non-normal variables are made of the equivalent normal, then there is calculation, specific analysis steps are as follows:

- Assume that the initial value of the common parameters.

- Set the initial value of checking point $X_{i}$, this step cannot be omitted for the current linear state equation.

- Calculate $\left.\frac{\partial \mathrm{g}}{\partial X_{i}}\right|_{p^{\circ}}$, if $\left.\frac{\partial \mathrm{g}}{\partial X_{i}}\right|_{p^{\circ}}<0, \beta_{i}^{-}$is calculated according to the type of the random variable division and calculation formula has been given; if $\left.\frac{\partial \mathrm{g}}{\partial X_{i}}\right|_{p^{\prime}}>0$, calculate $\beta_{i}^{+}$.

- Calculate $\sigma_{X_{i}}$ according to formula $\sigma_{X_{i}}=\frac{\beta_{i}^{ \pm} \sigma_{X_{i}}}{\beta}$.

- Calculate direction $\operatorname{cosine} \cos \theta_{X_{i}^{\prime}}$.

- Calculate $X_{i}^{*}$ according to $X_{i}^{*}=\mu_{X_{i}}+\beta \sigma_{X_{i}^{\prime}} \cos \theta_{X_{i}^{\prime}}$. 
- Taking the obtained coefficient of back to the limit equation $g\left(x_{1}^{*}, x_{2}^{*}, \ldots, x_{i}^{*}, \ldots x_{n}^{*}\right)=0$ to obtain $\beta$.

- If the value obtained in step 7 is close or equal to the assumed initial value of the step 1, end the iteration, the value of $\beta$ is the finial value, otherwise take the $\beta$ back into calculation until meeting the demand.

\section{Reliability ANALysis of ReINFORCED CONCRETE BEAM AFTER FIRE}

Take the beam with rectangular section as an example. For the fire side of beam is mostly on bottom surface during the process of temperature rising, the temperature of top side and inside increase a little. Because of this factor, the shear bearing capacity of the beam can be neglected. That is to say, the calculation of remaining bearing capacity of the RC beam after high temperature is mainly the bending bearing capacity, the following example is to explain the detail of the calculation process.

Assume that reinforced concrete beam member cross section size is $500 \mathrm{~mm} \cdot 1200 \mathrm{~mm}$. Stress analysis results: Under the action of dead load: The bending moment in the middle is: $\mathrm{M}_{0}=477 \mathrm{kN} \cdot \mathrm{m}$; the bending moment of both ends are: $M_{A}=970 \mathrm{kN} \cdot \mathrm{m}, \mathrm{MB}=688 \mathrm{kN} \cdot \mathrm{m}$; Under the action of live load: The bending moment in the middle is: $\mathrm{M}_{0}=110 \mathrm{kN} \cdot \mathrm{m}$. the bending moment of both ends are: $M_{A}=204 \mathrm{kN} \cdot \mathrm{m}$, $M_{B}=177 \mathrm{kN} \cdot \mathrm{m}$. The thickness of the protective layer is $40 \mathrm{~mm}$, Section effective height: $h_{0}=1147.5 \mathrm{~mm}$, the cross section is shown in Fig. 1.

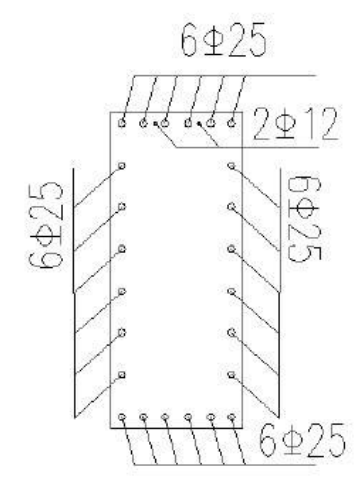

Figure 1. The beam section

Thermal analysis is carried out by means of finite element analysis software ANSYS, using solid70 unit. Heating up curve is the international standard heating curve, conduct the nonlinear transient analysis. Based on continuous simulation and comparison, it is found that when $\mathrm{t}=3240 \mathrm{~s}(2.5 \mathrm{~h})$, the temperature is 541.27 in the point $(0.046,0.046)$ in the component section temperature field, which is closed to $\mathrm{T}=541.1^{\circ} \mathrm{C}$. So the equivalent time $\mathrm{t}$ is set by fire of the component is 3240s in the example, moreover, the component section temperature field distribution is shown in Fig. 2.

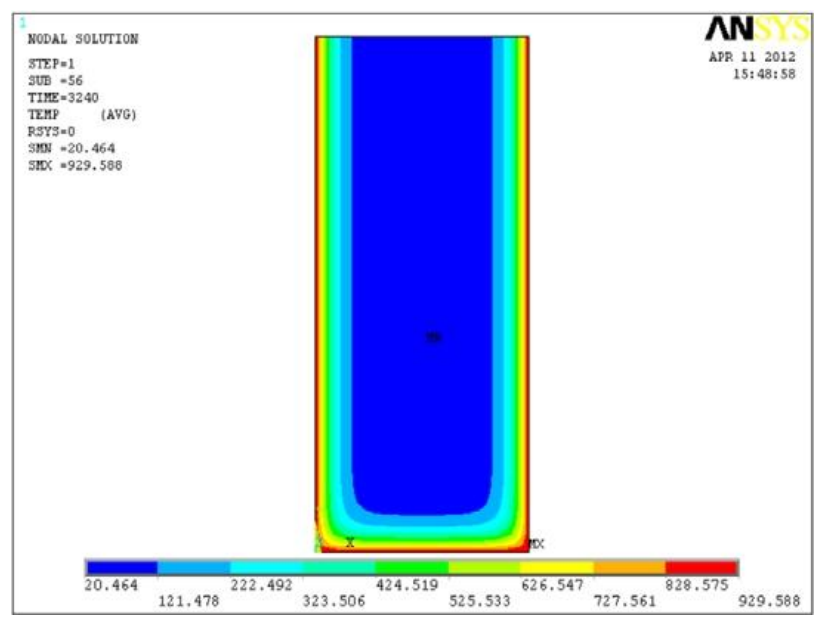

Figure 2. The component section temperature field distribution

In this part, the strength of the beam cross section reduction factor is calculated by using hierarchical method. Firstly, the section of component is divided by rectangular grid cell and using the highest temperature value in the lower right corner coordinates as the highest temperature experienced by the grid cell. According to temperature data of each node which is given by ANSYS software, the temperature value of each unit is known. Then the unit concrete strength reduction factor $\frac{\varphi_{\overline{C T i}}}{=}=\frac{f_{c r}(T)}{f_{c}}$ is obtained by calculating units' concrete strength ${ }^{f_{c c}(T)=\frac{f_{c}}{1+2.4(T-20)^{6} \times 10^{-17}}}$. Finally the entire section unit intensity reduction factor is weighted averagely, the whole cross section of concrete intensity

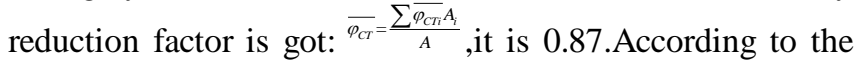
statistical parameter analysis and the limit state equation of beams in the third quarter, the reliability indicators of beam after fire is 3.82 by the improved checking point method, which meets the requirements of not less than 3.2.

\section{CONCLUSION}

The reduction factor of the components intensity is got by the layered method, the component materials, geometric parameters and calculation mode are analysis statistically, the residual bearing capacity of concrete members is simulated after fire by ANSYS. Finally, the reliability of beam structures is calculated after fire by the improved checking point method. It not only makes reference to construction safety analysis after fire, but also makes further reference for structure reliability analysis and evaluation.

\section{ACKNOWLEDGEMENTS}

The work was funded by Shaanxi Province National Science Foundation for Young Scholars (2012JQ7018).

\section{REFERENCES}

[1] Lili Bai," The reliability analysis of reinforced concrete's structural members after fire "[D], Harbin: Harbin engineering university, Ph.D. Thesis, 2008: 11-66.

[2] Bo Wu," The mechanical properties of reinforced concrete structures after fire "[M],Beijing: Science press, 2003:1-40. 
[3] Guhua Li, Lingyun Feng, Shenge Zheng," Performance study of concrete after high temperature and its materials " [J], Sichuan construction science research, 1991,2:1-5.

[4] Jinxin Gong, Guofan Zhao, Lin Liu," Reinforced concrete axial compression member after reinforcement of reliability analysis " [J], Building structure, 2000,30(3):29-33.

[5] Wei Li, Zhenhai Guo," The experimental study of strength and deformation characteristics of concrete under high temperature" [J], Journal of building structures, 1993,14(1):8-16.

[6] Tian Li, Xila Li," The durability of concrete structure analysis and design " [M],Beijing: Science press, 1999.
[7] Yongjun Liu," The fire response of reinforced concrete structures, numerical simulation and software development " [D],Dalian: Dalian university of technology,2002:51-62.

[8] Huilan Yao, Chunfen Jia, Huiqiang Zhi, Shichang Lu," The application of ANSYS in the structure response analysis " [J], Journal of hebei institute of building science and technology,2006,23(1):4952 .

[9] Xilong Tang, Kebo Lin, Meixin Ye," The mechanical properties research of reinforced concrete members under high temperature " [J], Test technology and the testing machine, 2006,46(1):35-38.

[10] Bin Li, "The reliability analysis of the numerical simulation of concrete structure by fire " [D],Kunming: Kunming university of science and technology, 2008. 